\title{
Sonic Hedgehog Pathway Contributes to Gastric Cancer Cell Growth and Proliferation
}

\author{
Jianhua Wan, ${ }^{1, \star}$ Ji Zhou, ${ }^{2, *}$ Hailong Zhao, Mei Wang,, Zhuanqin Wei, Hongyan Gao, \\ Yongzhong Wang, ${ }^{3}$ and Hongjuan Cui ${ }^{1}$
}

\begin{abstract}
The Sonic Hedgehog (Shh) signaling pathway is commonly activated in gastrointestinal cancer. However, our understanding of the Shh pathway in gastric cancer remains limited. Here we examined the effects of cyclopamine, a specific inhibitor of the Shh signaling pathway, on cell growth and proliferation in gastric primary cancer cells GAM-016 and the MKN-45 cell line. The results showed that the Shh signaling molecules SHH, PTCH, SMO, GLI1, and GLI2 were intact and activated in both types of cells. Furthermore, we observed that cyclopamine inhibited gastric cancer cell proliferation through cell cycle arrest and apoptosis. An in vivo study using NOD/SCID mouse xenografts demonstrated that cyclopamine significantly prevented tumor growth and development. Our study indicated that Shh signaling pathway could promote gastric cancer cell proliferation and tumor development, and blocking this pathway may be a potential strategy in gastric cancer treatment.
\end{abstract}

Key words: apoptosis; cell cycle arrest; cell proliferation; cyclopamine; primary gastric cancer; Sonic Hedgehog

\section{Introduction}

$\mathbf{G}$ ASTRIC CANCER IS ONE OF THE most commonly diagnosed cancers, leading to approximately 800,000 deaths every year worldwide. Despite some advances in the treatment of gastric cancer, the prognosis of patients remains poor, with a 5-year survival rate of less than $5 \% .{ }^{1}$ Therefore, novel therapeutic strategies are urgently required.

Sonic Hedgehog (Shh), as a member of the Hedgehog family, was originally identified by its homology to the Drosophila melanogaster segment polarity gene Hedgehog, and the Shh signaling pathway is highly conserved in mammals and other vertebrate species. ${ }^{2,3}$ When it binds to its receptor Patched 1 (PTCH1), SHH can enhance the activity of the transmembrane protein Smoothened (SMO), ${ }^{4}$ and the GLI transcription factor subsequently regulates the expression of target genes that control cell growth, survival, and differentiation in various tissues. ${ }^{5-7}$

Accumulating evidence has shown that the Shh signaling pathway is activated in several cancer types, including neuroblastoma, colon cancer, and small cell lung cancer. ${ }^{8-15}$ Moreover, recent data have proven that the Shh pathway is correlated with the initiation of gastrointestinal cancers. ${ }^{16-18}$ Therefore, more understanding of this pathway in cancer de- velopment will contribute to treatment of gastric cancers. In this study, we found that cyclopamine can inhibit cell proliferation and tumor growth by blocking the Shh signaling pathway in gastric cancer.

\section{Materials and Methods}

Primary cell source

Primary human gastric cancer specimens were obtained with patients' written consent from Daping Hospital, the Third Military Medical University, China. This project was approved by the Ethical Committee of Southwest University, China. Animal studies were conducted with approval from the Institution's Animal Care and Use Committees at Southwest University.

\section{Cell culture}

Human primary gastric cancer specimens were dissociated and digested into single-cell suspension by collagenase and DNase cocktail, and cells were grown in Neuro-basal culture medium (Invitrogen, Carlsbad, CA) supplemented with $20 \mathrm{ng} / \mathrm{L}$ epidermal growth factor and $20 \mathrm{ng} / \mathrm{L}$ b-fibroblast growth factor (Sigma, Magdeburg, Germany). ${ }^{19}$ We refer

\footnotetext{
${ }^{1}$ State Key Laboratory of Silkworm Genome Biology, Southwest University, Chongqing, China.

${ }^{2}$ Department of Neurosurgery, Daping Hospital, Third Military Medical University, PLA, Chongqing, China.

${ }^{3}$ Department of Radiology, Fourth Hospital of Hebei Medical University, Shijiazhuang, Hebei Province, China

*These authors contributed equally to this work.
} 
to the primary gastric cancer cells as GAM-016, based on the patient's sex and case number. MKN-45 cell line from the ATCC was grown in RPMI 1640 supplemented with $10 \%$ fetal bovine serum (Invitrogen). Both lines were cultured at $37^{\circ} \mathrm{C}$ in $5 \% \mathrm{CO}_{2}$ humidified incubator and were subcultured at the split ratio of $1: 3$ every 3 days.

\section{Cyclopamine treatment or BrdU incubation}

Since tomatidine is structurally similar to cylopamine but does not inhibit the Shh pathway, we used tomatidine as the experimental control to investigate if specific inhibition of the Shh pathway by cyclopamine was the primary cause of decreased cell proliferation and tumor growth. ${ }^{20,21}$

Cyclopamine was dissolved in dimethyl sulfoxide (DMSO) as a $10 \mathrm{mM}$ stock solution. GAM-016 and MKN45 cells were treated with $20 \mu \mathrm{M}$ cyclopamine or tomatidine. ${ }^{22}$ Micrographs of cell morphology were taken with an Olympus microscope (80i, Tokyo, Japan). Cell growth number was analyzed by trypan blue exclusion assay. ${ }^{23}$

The thymidine analog 5-bromo-2-deoxyuridine (BrdU; Sigma) was added to the stock solution, which was prepared in phosphate-buffered saline (PBS) at $10 \mathrm{mM}$ and diluted $1000 \times$ for the working solution. After incubation for $45 \mathrm{~min}$ in the culture medium, the cells were ready for BrdU antibody staining.

\section{Immunofluorescent staining}

Cells were grown on cover slips and treated with either tomatidine or cyclopamine. After treatment, cells were successively washed with PBS, fixed in $4 \%$ paraformaldehyde (Beyotime, Chongqing, China) for $15 \mathrm{~min}$, and permeabilized with $0.3 \%$ Triton X-100 for $5 \mathrm{~min}$. Cells were blocked with $10 \%$ horse serum for $1 \mathrm{~h}$ at $37^{\circ} \mathrm{C}$, incubated with a primary antibody for $2 \mathrm{~h}$ at room temperature, followed by the appropriate secondary antibody for $1 \mathrm{~h}$, and $300 \mathrm{nM}$ 4',6-diamidino-2-phenylindole for counterstaining at room temperature. $^{24,25}$ Rat anti-BrdU (ab6326; Abcam, Cambridge, United Kingdom) 1:200, goat anti-GLI2 (N-20; Santa Cruz Biotechnology, Shanghai, China) 1:50, goat anti-SHH (N-19; Santa Cruz Biotechnology) 1:50, goat anti-GLI1 (N16; Santa Cruz Biotechnology) 1:50, goat anti-patched (C20; Santa Cruz Biotechnology) 1:50, and rabbit anti-SMO (H-300; Santa Cruz Biotechnology) 1:50 were used as primary antibodies. Alexa Fluor 488 goat anti-rat $\operatorname{IgG}(\mathrm{H}+\mathrm{L})$ 1:600, Cy3-labeled donkey anti-goat IgG, or Cy3-labeled goat antirabbit IgG 1:500 (Beyotime) was used as the secondary antibody. A Nikon microscope with Image-Pro Plus software was used to record and analyze the fluorescent signaling images (Holdtecs Technology Co., Ltd, Chengdu, China).

\section{MTT assay}

Cell growth curve was determined by 3-(4,5-dimethylthiazol-2-yl)-2,5-diphenyl tetrazolium bromide (MTT) assay. Cells were planted onto a 96-well plate at 400 cells/well overnight. After incubation with cyclopamine or tomatidine for a certain time period, $20 \mu \mathrm{L}$ of MTT $(5 \mathrm{mg} / \mathrm{mL}$ MTT in PBS; Sigma) was added to each well, and the cells were then incubated at $37^{\circ} \mathrm{C}$ for $2 \mathrm{~h}$. DMSO (200 $\mu \mathrm{L}$ per well; Sigma) was added to dissolve the cell. The absorbance was measured at a wavelength of $570 \mathrm{~nm}$ after shaking for $10 \mathrm{~min}^{26-28}$

\section{Cell cycle assay}

Cells were collected and fixed in $70 \%$ ethanol. After being stained with propidium iodide (PI; Beyotime), the cells were analyzed by flow cytometry (BD BioSciences, Franklin Lakes, NJ). The data were analyzed by Cell Quest Pro (BD BioSciences) and ModFit 3.1 (Verity Software, Topsham, United Kingdom).

\section{Apoptosis analysis}

Exponentially growing cells at $70 \%-80 \%$ confluence were treated with $20 \mu \mathrm{M}$ cyclopamine or tomatidine. Adherent and floating cells were collected by centrifugation, and washed with ice-cold PBS. Apoptotic cell was determined by staining with PI and 7-aminoactinomycin D (Beyotime), followed by flow cytometry (BD Biosciences) analysis.

\section{Reverse-transcription polymerase chain reaction assay}

Cells were treated with either cyclopamine or tomatidine for 7 days and harvested. Total RNA was extracted by Trizol method

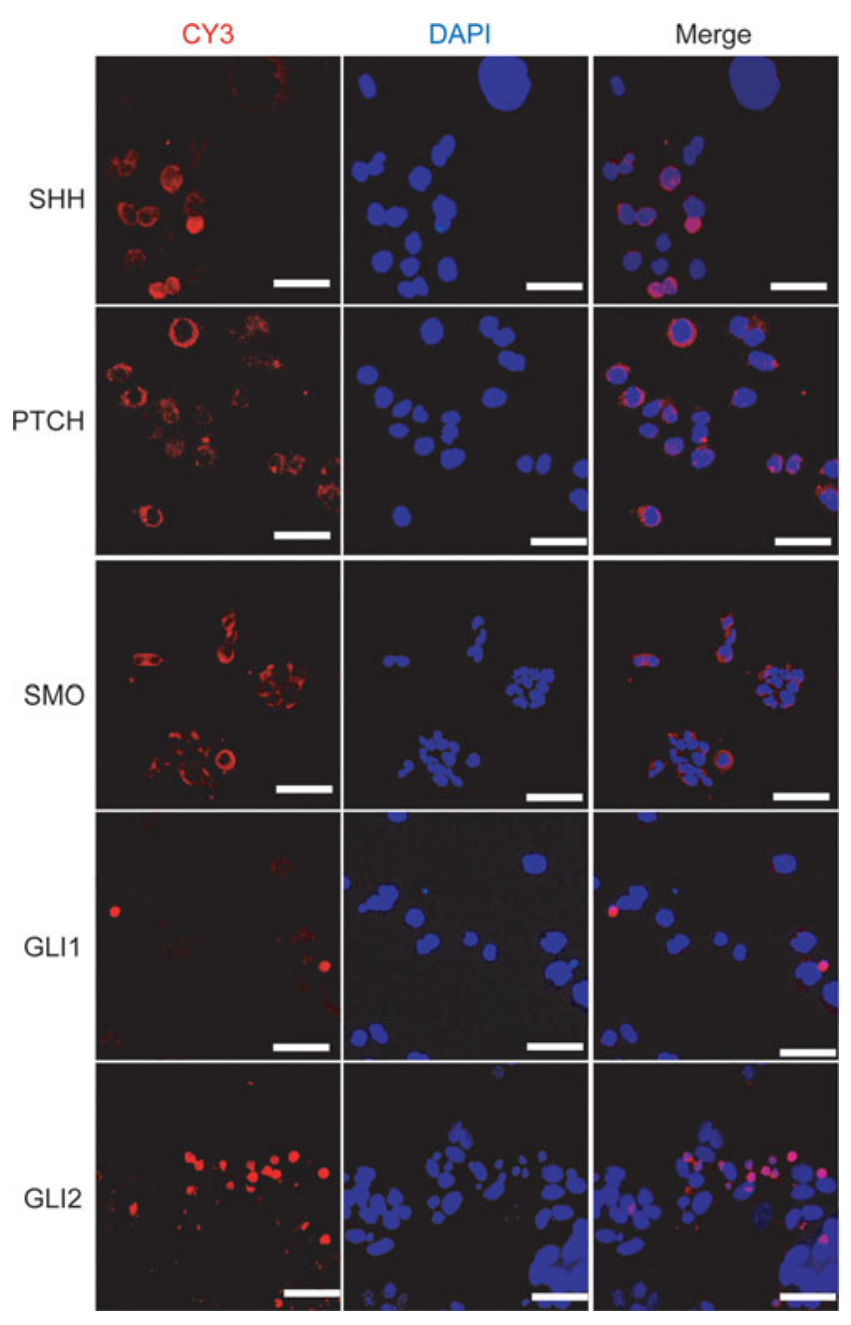

FIG. 1. The Sonic Hedgehog (Shh) signaling pathway components are detected in primary gastric cancer cells. Immunofluorescent staining was manipulated to investigate the expression of SHH, PTCH, SMO, GLI1, and GLI2 in human primary gastric cancer cells (red), and the nuclei were counterstained with 4',6-diamidino-2-phenylindole (DAPI; blue). Scale bar, $100 \mu \mathrm{m}$. 

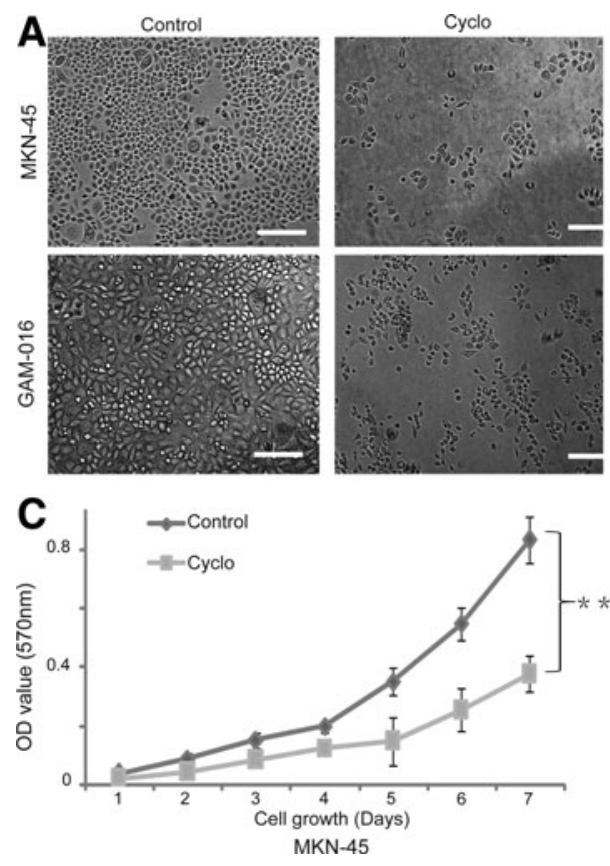
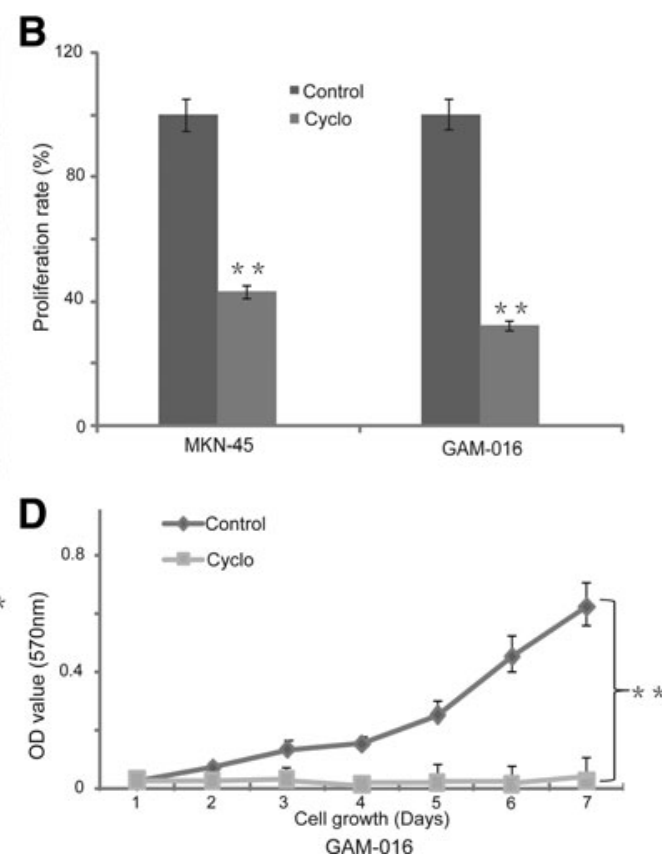

FIG. 2. The Shh pathway is required for the cell growth and proliferation in primary gastric cancer cells and cell line. (A) The primary gastric cancer cells (GAM-016) and MKN-45 cells were treated with $20 \mu \mathrm{M}$ tomatidine or cyclopamine for 4 days. Cell morphology was observed. Scale bar, $100 \mu \mathrm{m}$. (B) The proliferation rate of GAM016 and MKN-45 cells was determined by cell number; the proliferation rate of tomatidine-treated cells was designated as $100 \%$. (C, D) 3-(4,5-Dimethylthiazol-2yl)-2,5-diphenyl tetrazolium bromide (MTT) assay was conducted daily from 1 to 7 days. Data are presented as mean \pm SD. ${ }^{*} p<0.05$; $* * p<0.01$. and reverse transcribed into cDNA by M-MLV (Promega, Madison, WI). Real-time polymerase chain reaction (PCR) was performed using a SYBR Green PCR Master Mix (Takara) with primers against Shh, Ptch, Gli1, Gli2, and CCND1. All primer pairs were verified by melting curve analysis following quanti- tative reverse-transcription (RT)-PCR, with each primer pair showing a single desired amplification peak. ${ }^{29}$ The individual values were normalized to that of the tomatidine control, and the ratio of the relative expression levels over that of the vehicle-treated cells was calculated.

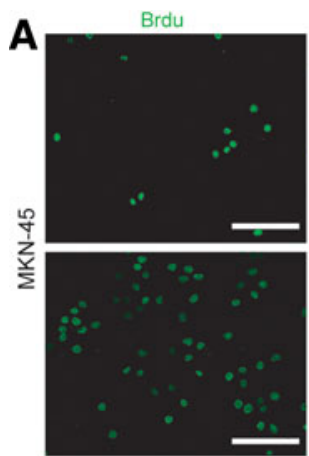

B

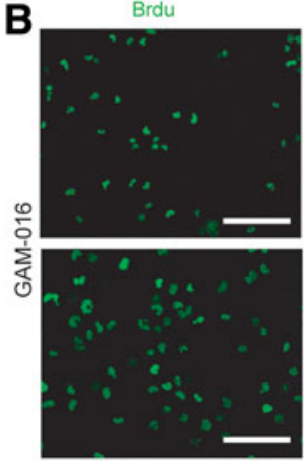

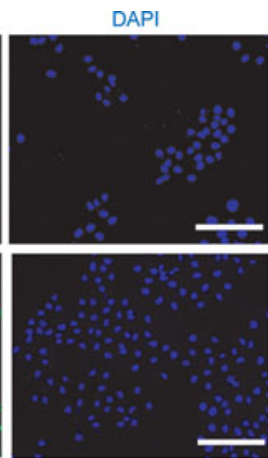

DAPI

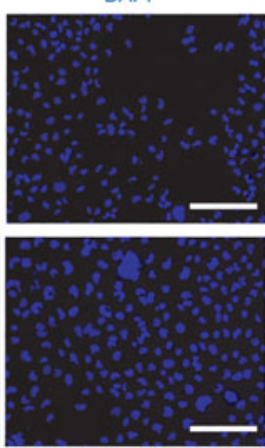

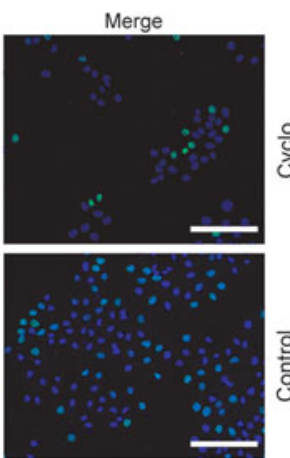

Merge

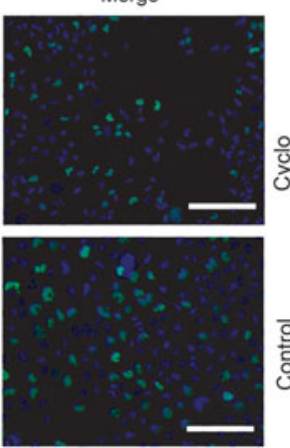

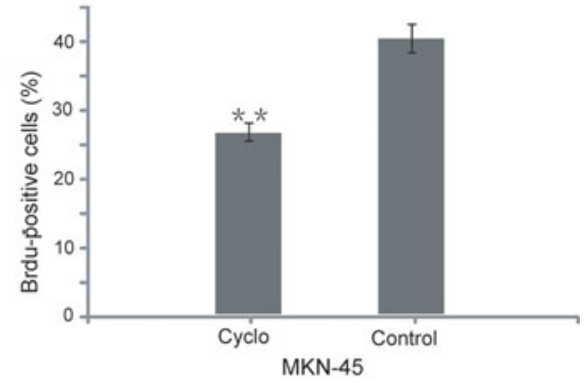

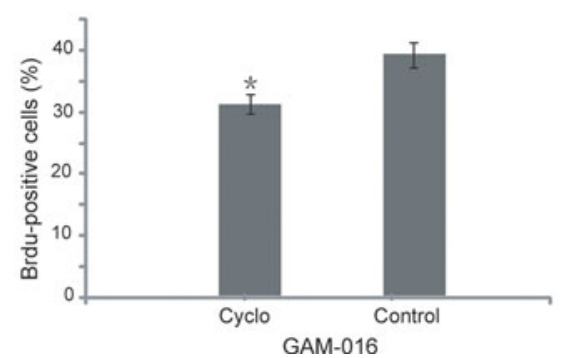

FIG. 3. 5-Bromo-2-deoxyuridine (BrdU) immunofluorescent staining was accomplished in GAM-016 and MKN-45 cells. (A, B) Cells were grown on coverslips and treated with $20 \mu \mathrm{M}$ tomatidine or cyclopamine for 4 days. After treatment, BrdU was added and cells were incubated for another $45 \mathrm{~min}$. Cells were stained with an antibody against BrdU (green), and counterstained with DAPI (blue). The percentage of BrdU-positive cells was calculated. Cells were counted from at least five randomly selected fields. Data are presented as mean \pm SD. ${ }^{*} p<0.05 ; * * p<0.01$. Scale bar, $100 \mu \mathrm{m}$. 


\section{Xenograft tumor model}

One million primary gastric cancer cells were injected subcutaneously into flanks of 4-week-old NOD/SCID female mice (Beijing Laboratory Animal Research Center, Beijing, China). After 7 days of tumor growth, the mice were treated with cyclopamine $(0.4 \mathrm{mg}$ intraperitoneally) for 7 days. Tumor volumes were calculated daily based on caliper measurements of tumor length and width (volume = tumor length $\times$ width $\left.^{2} \times 0.5236\right)$ after treatment. Then the mice were sacrificed, and the tumors were excised and weighed.

\section{Statistical analysis}

Assays were set up in triplicate, and the results are presented as mean \pm SD. Variance between the experimental groups was determined by two-tailed $t$-test, and $p<0.05$ was considered statistically significant.

\section{Results}

The Shh signaling pathway was intact and activated in primary gastric cancer cells

To investigate the role of the Shh signaling pathway, we detected the expression of Shh signaling pathway compo- nents in GAM-016 cells by immunofluorescent staining. As expected, SHH, PTCH, and SMO staining patterns were observed on the membrane, while GLI1 and GLI2 were principally localized in the nucleus (Fig. 1). These observations demonstrated that the Shh signaling pathway was intact and activated in primary gastric cancer cells.

\section{The Shh pathway was required for cell growth and proliferation in GAM-016 and MKN-45 cells}

Next, we investigated whether the Shh signal pathway is required in cancer cell growth and proliferation. The GAM-016 and $\mathrm{MKN}-45$ cells were used in this experiment. Cyclopamine, a steroidal alkaloid that specifically recognizes the Shh signaling pathway through direct interaction with SMO, was used to block the Shh pathway. ${ }^{30-32}$ Treatment with cyclopamine caused a significant decrease in the number of viable cells compared with tomatidine-treated cells (Fig. 2A). The cell growth number was reduced more than $70 \%$ compared with the control in GAM-016 cells (Fig. 2B). Similarly, cyclopamine treatment also reduced $55 \%$ of cell growth in MKN-45 cell line (Fig. 2B). The MTT assay also showed that cyclopamine significantly inhibited cell growth. GAM-016 cells were more sensitive to the treatment than MKN-45 cells (Fig. 2C, D).
FIG. 4. Inhibition of the Shh signaling pathway induced cell cycle arrest and apoptosis. (A, B) Cells were treated with $20 \mu \mathrm{M}$ tomatidine or cyclopamine for 4 days, and the cell cycle was analyzed by fluorescent activated cell sorting. (C) The CCND1 mRNA expression level was determined by reverse-transcription polymerase chain reaction (RT-PCR) analysis. Tomatidine treatment was a control. (D) Apoptosis induced by cyclopamine was determined by flow cytometry. Data are presented as mean \pm SD. $* p<0.05$; ** $p<0.01$. (E, F) The mRNA expression level of major Shh signaling pathway molecules (such as Shh, Ptch, Gli1, and Gli2) was detected by RT-PCR analysis after 7 days of treatment at $10 \mu \mathrm{M}$ cyclopamine. Tomatidine served as a control.
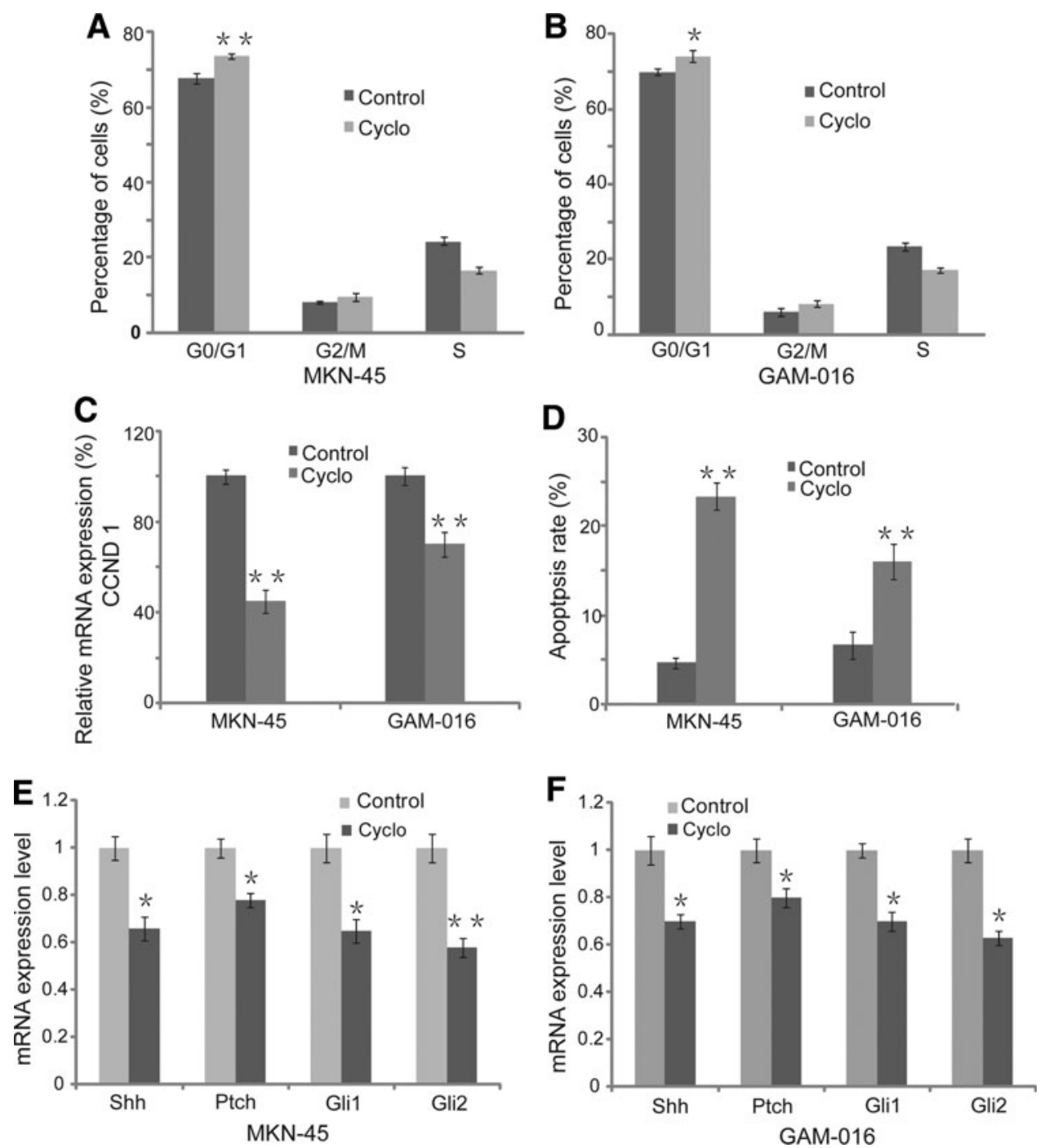


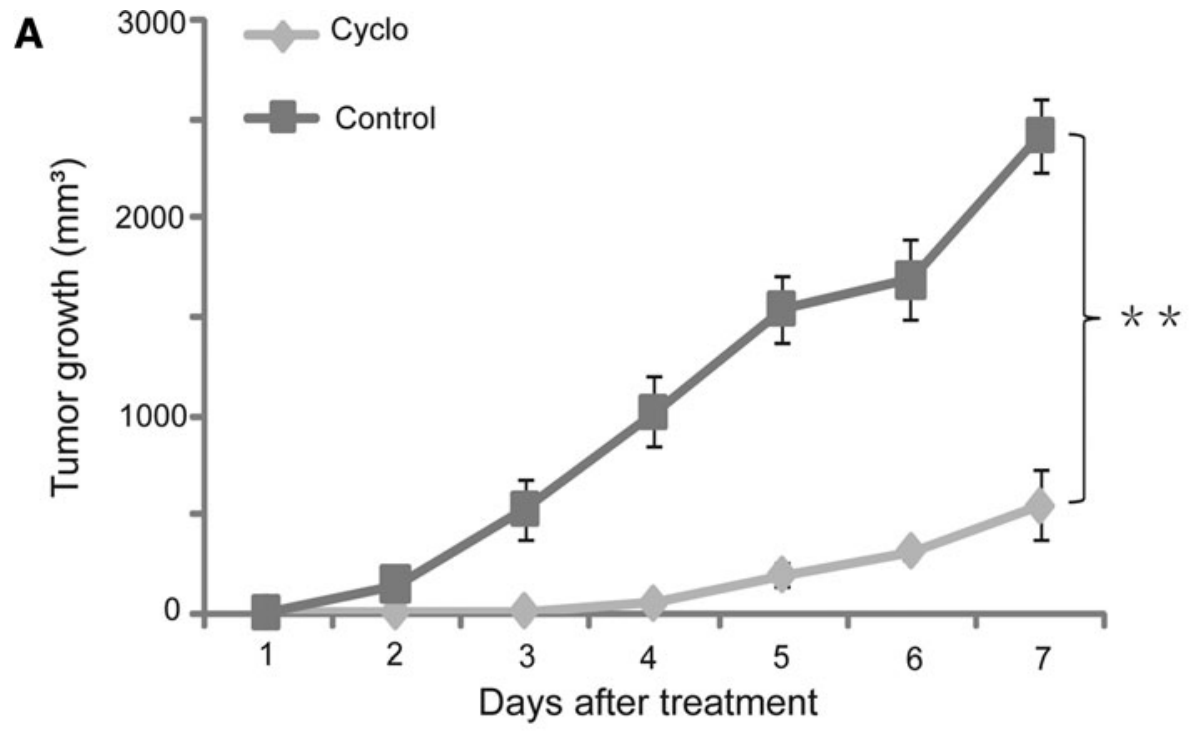

B

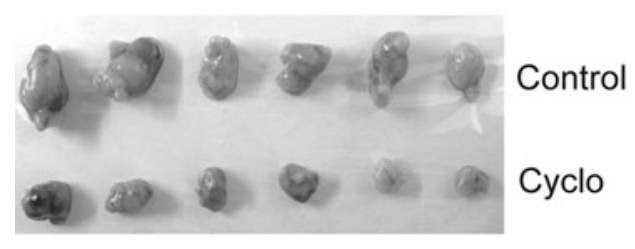

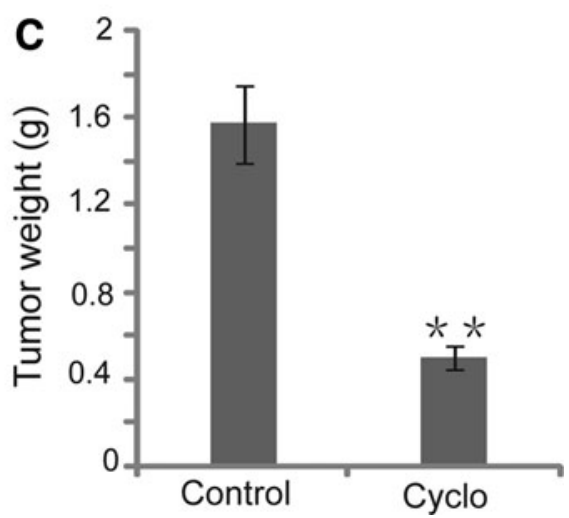

FIG. 5. Blockage of the Shh signaling pathway attenuated tumor growth in NOD/SCID mice xenografts model. (A) Tumor growth curve at 7 days after cyclopamine treatment (0.4 mg intraperitoneally). (B) Photograph of tumors resected from NOD/SCID mice. (C) Quantitative analysis of tumor weight. Data are presented as mean \pm SD. ${ }^{*} p<0.05 ; * * p<0.01$.
BrdU staining assay was also carried out to further confirm that there was a dramatic inhibition in cell proliferation with cyclopamine treatment in both GAM-016 and MKN-45 cells (Fig. 3A, B).

\section{Cell cycle arrest and apoptosis were induced by inhibition of the Shh signaling pathway}

To elucidate the mechanism of Shh-mediated cell survival and proliferation, the cell cycle and apoptosis were tested and analyzed. We observed that there was a significant reduction in the proportion of the $\mathrm{S}$ phase cells $(23.5 \%$ to $17.3 \%$ and $24.5 \%$ to $16.7 \%$ in MKN-45 and GAM-G16 cells, respectively), while a simultaneous increase occurred in both G0/G1 and G2/M phase cells after cyclopamine treatment (Fig. 4A, B). These data revealed that cyclopamine treatment blocked the G0/G1-S transition and induced G0/ G1 cell cycle arrest in GAM-016 and MKN-45 cells. RTPCR analysis showed that the mRNA level of CCND1 was dramatically decreased after cyclopamine treatment, which caused cell cycle arrest at the G1 phase. In addition, we found that cyclopamine also induced cell apoptosis in GAM-016 and MKN-45 cells (Fig. 4C, D). Furthermore, decreased expression of major Shh signaling pathway molecules was observed using RT-PCR, suggesting that the Shh signaling pathway plays a pivotal role in promoting the survival of gastric cancer cells (Fig. 4E, F).

\section{Tumor growth in NOD/SCID mice was attenuated by blockage of the Shh signaling pathway}

We established a NOD/SCID mouse xenograft model to test the function of the Shh pathway in tumor growth and development. After 10-day treatment with cyclopamine, the tumor growth in SCID mice decreased nearly $80 \%$ compared to the control group (Fig. 5A). The tumor weight in the cyclopamine group was dramatically lower than in the control group (Fig. $5 \mathrm{C})$. We also observed that there was no significant difference in body weight between the treatment and the control group (data not shown), which suggests that cyclopamine treatment may have minimal side effect or toxicity to the mice. Therefore, blocking the Shh signaling pathway might be a promising strategy for gastric cancer treatment.

\section{Discussion}

Although several clinical and pathologic studies have demonstrated that $\mathrm{SHH}$ and the expression of its signaling cascade proteins are associated with the progression of gastric cancer, ${ }^{33-36}$ the definitive role of the Shh pathway in gastric cancer remains unknown and requires further investigation. ${ }^{37}$ Previous studies have shown that the activation of the Shh pathway can promote cancer cell survival and cell cycle progression via up-regulation of $\mathrm{Bcl} 2^{34,38}$ and expression of G1-phase cyclins, respectively, ${ }^{39,40}$ 
especially Cyclin-D, advancing cell cycle from G1 phase to $S$ phase. ${ }^{41,42}$ In the current study, we used a primary gastric cancer cell suspension and the MKN-45 cell line to clarify the function of Shh signaling in gastric cancer in vitro and in vivo.

Our results showed that the blockage of Shh signaling by the specific inhibitor cyclopamine could reduce CCND1 expression and induce G0/G1 arrest, which is consistent with previous studies. ${ }^{40,41}$ We presented results from a tomatidine control, which supported cyclopamine being specific to the Shh pathway. We also found that cyclopamine treatment induced cell apoptosis in gastric cancer cells and inhibited tumor growth, which had previously been observed in melanomas and cholangiocarcinomas. ${ }^{43-45}$ Thus, it was illustrated that Shh signaling plays a crucial role in cell growth, survival, and proliferation in gastric cancer cells.

In conclusion, our results demonstrated that the Shh signaling pathway is directly correlated with gastric cancer cell proliferation and tumor growth. Therefore, it is possible that the multiplication and development of gastric cancer cells could be retarded by cyclopamine as a specific inhibitor by blocking the Shh signaling pathway. This suggests that the Shh pathway could be a promising therapeutic target in gastric cancer treatment.

\section{Acknowledgments}

We appreciate Qinrui Wang from the Australian National University for her kind editing of this manuscript. This study was supported by the National Basic Research Program of China (No. 2012cb114603), the Research Fund for the Doctoral Program of High Education of China (20130182110003), and the Fundamental Research Funds for the Central Universities (XDJK2013B020). Author contributions: J.W. performed most of the experiments; J.Z. was in charge of drafting the manuscript; H.Z. participated in the MTT assay; Z.W. and H.G. participated in the primary cell culture; Y.W. was in charge of the primary tumor specimen; Q.X. took part in the experimental design; and H.C. was responsible for the whole project. All authors read and approved the final manuscript.

\section{Author Disclosure Statement}

No competing financial interests exist.

\section{References}

1. Ito $\mathrm{H}$, Inoue $\mathrm{H}$, Ikeda $\mathrm{H}$, et al. Surgical outcomes and clinicopathological characteristics of patients who underwent potentially noncurative endoscopic resection for gastric cancer: a report of a single-center experience. Gastroenterol Res Pract. 2013;2013:427405.

2. Fietz MJ, Concordet JP, Barbosa R, et al. The Hedgehog gene family in Drosophila and vertebrate development. Dev Suppl. 1994;1994:43-51.

3. Varjosalo M, Taipale J. Hedgehog signaling. J Cell Sci. 2007;120:3-6.

4. Ingham PW. Hedgehog signaling. Cold Spring Harb Perspect Biol. 2012;4. pii:a011221.

5. Taipale J, Beachy PA. The Hedgehog and Wnt signalling pathways in cancer. Nature. 2001;411:349-354.

6. Varnat F, Duquet A, Malerba M, et al. Human colon cancer epithelial cells harbour active HEDGEHOG-GLI signalling that is essential for tumour growth, recurrence, metastasis and stem cell survival and expansion. EMBO Mol Med. 2009;1:338-351.

7. Wickström M, Dyberg C, Shimokawa T, et al. Targeting the Hedgehog signal transduction pathway at the level of GLI inhibits neuroblastoma cell growth in vitro and in vivo. Int J Cancer. 2013;7:1516-1524.

8. Xu L, Wang X, Wan J, et al. Sonic Hedgehog pathway is essential for neuroblastoma cell proliferation and tumor growth. Mol Cell Biochem. 2012;364:235-241.

9. Li T, Wang L, Ke XX. DNA-damaging drug-induced apoptosis sensitized by $\mathrm{N}$-myc in neuroblastoma cells. Cell Biol Int. 2012;36:331-337.

10. Hooper JE, Scott MP. Communicating with Hedgehogs. Nat Rev Mol Cell Biol. 2005;6:306-317.

11. Riobo NA, Manning DR. Pathways of signal transduction employed by vertebrate Hedgehogs. Biochem J. 2007; 403:369-379.

12. Rubin LL, de Sauvage FJ. Targeting the Hedgehog pathway in cancer. Nat Rev Drug Discov. 2006;5:1026-1033.

13. Watkins DN, Berman DM, Burkholder SG, et al. Hedgehog signaling within airway epithelial progenitors and in smallcell lung cancer. Nature. 2003;422:313-317.

14. Mao L, Xia YP, Zhou YN, et al. A critical role of Sonic Hedgehog signaling in maintaining the tumorigenicity of neuroblastoma cells. Cancer Sci. 2009;100:1848-1855.

15. Jeng KS, Sheen IS, Jeng WJ, et al. Blockade of the Sonic Hedgehog pathway effectively inhibits the growth of hepatoma in mice: an in vivo study. Oncol Lett. 2012;4:11581162.

16. Dosch JS, Pasca di Magliano M, Simeone DM. Pancreatic cancer and Hedgehog pathway signaling: new insights. Pancreatology. 2010;10:151-157.

17. Mao J, Kim BM, Rajurkar M, et al. Hedgehog signaling controls mesenchymal growth in the developing mammalian digestive tract. Development. 2010;137:1721-1729.

18. Song Z, Yue W, Wei B, et al. Sonic Hedgehog pathway is essential for maintenance of cancer stem-like cells in human gastric cancer. PLoS One. 2011;6:e17687.

19. Yang LQ, Cui HJ, Wang Z. Loss of negative feedback control of nuclear factor-kappa B2 activity in lymphocytes leads to fatal lung inflammation. Am J Pathol. 2010;176:26462657.

20. Heretsch P, Buttner A, Tzagkaroulaki L, et al. Exo-cyclopamine - a stable and potent inhibitor of Hedgehog-signaling. Chem Commun (Camb). 2011;47:7362-7364.

21. Wu X, Cai ZD, Lou LM, et al. The effects of inhibiting Hedgehog signaling pathways by using specific antagonist cyclopamine on the chondrogenic differentiation of mesenchymal stem cells. Int J Mol Sci. 2013;14:5966-5977.

22. Yauch RL, Gould SE, Scales SJ, et al. A paracrine requirement for Hedgehog signalling in cancer. Nature. 2008;455: 406-410.

23. Li T, Zhao BC, Ke XX, et al. Essential role for p53 and caspase-9 in DNA damaging drug-induced apoptosis in neuroblastoma IMR32 cells. DNA Cell Biol. 2011;30: 1045-1050.

24. Ng JC. A quantum dot-immunofluorescent labeling method to investigate the interactions between a crinivirus and its whitefly vector. Front Microbiol. 2013;4:77.

25. van de Werken C, Jahr H, Avo Santos M, et al. A universal method for sequential immunofluorescent analysis of chromatin and chromatin-associated proteins on chromosome spreads. Chromosome Res. 2013;21:475-489. 
26. Buch K, Peters T, Nawroth T, et al. Determination of cell survival after irradiation via clonogenic assay versus multiple MTT assay - a comparative study. Radiat Oncol. 2012;7:1.

27. Li Y, Huang W, Huang S, et al. Screening of anti-cancer agent using zebrafish: comparison with the MTT assay. Biochem Biophys Res Commun. 2012;422:85-90.

28. Stockert JC, Blazquez-Castro A, Canete M, et al. MTT assay for cell viability: Intracellular localization of the formazan product is in lipid droplets. Acta Histochem. 2012; 114:785-796.

29. Zhu SQ, Li T, Tan J, et al. Bax is essential for death receptor-mediated apoptosis in human colon cancer cells. Cancer Biother Radiopharm. 2012;27:577-581.

30. Chen JK, Taipale J, Cooper MK, et al. Inhibition of Hedgehog signaling by direct binding of cyclopamine to Smoothened. Genes Dev. 2002;16:2743-2748.

31. Cooper MK, Porter JA, Young KE, et al. Teratogenmediated inhibition of target tissue response to Shh signaling. Science. 1998;280:1603-1607.

32. Incardona JP, Gaffield W, Kapur RP, et al. The teratogenic Veratrum alkaloid cyclopamine inhibits Sonic Hedgehog signal transduction. Development. 1998;125:3553-3562.

33. Anton Aparicio LM, Garcia Campelo R, Cassinello Espinosa $\mathrm{J}$, et al. Prostate cancer and Hedgehog signalling pathway. Clin Transl Oncol. 2007;9:420-428.

34. Bar EE, Chaudhry A, Farah MH, et al. Hedgehog signaling promotes medulloblastoma survival via Bc/II. Am J Pathol. 2007; 170:347-355.

35. Alinger B, Kiesslich T, Datz C, et al. Hedgehog signaling is involved in differentiation of normal colonic tissue rather than in tumor proliferation. Virchows Arch. 2009;454:369-379.

36. Daya-Grosjean L, Couve-Privat S. Sonic Hedgehog signaling in basal cell carcinomas. Cancer Lett. 2005;225:181-192.

37. Berman DM, Karhadkar SS, Maitra A, et al. Widespread requirement for Hedgehog ligand stimulation in growth of digestive tract tumors. Nature. 2003;425:846-851.

38. Bigelow RL, Chari NS, Unden AB, et al. Transcriptional regulation of bcl-2 mediated by the Sonic Hedgehog signaling pathway through gli-1. J Biol Chem. 2004;279:1197-1205.

39. Kenney AM, Rowitch DH. Sonic Hedgehog promotes G(1) cyclin expression and sustained cell cycle progression in mammalian neuronal precursors. Mol Cell Biol. 2000;20:90559067.

40. Komada M. Sonic Hedgehog signaling coordinates the proliferation and differentiation of neural stem/progenitor cells by regulating cell cycle kinetics during development of the neocortex. Congenit Anom (Kyoto). 2012;52:72-77.
41. Gartel AL, Serfas MS, Tyner AL. p21-negative regulator of the cell cycle. Proc Soc Exp Biol Med 1996;213:138-149.

42. Dhulipala VC, Welshons WV, Reddy CS. Cell cycle proteins in normal and chemically induced abnormal secondary palate development: a review. Hum Exp Toxicol. 2006;25:675-682.

43. Das S, Harris LG, Metge BJ, et al. The Hedgehog pathway transcription factor GLI1 promotes malignant behavior of cancer cells by up-regulating osteopontin. J Biol Chem. 2009;284:22888-22897.

44. Geng L, Cuneo KC, Cooper MK, et al. Hedgehog signaling in the murine melanoma microenvironment. Angiogenesis. 2007;10:259-267.

45. Kurita S, Mott JL, Cazanave SC, et al. Hedgehog inhibition promotes a switch from Type II to Type I cell death receptor signaling in cancer cells. PLoS One. 2011;6:e18330.

Address correspondence to: Hongjuan Cui, PhD

State Key Laboratory of Silkworm Genome Biology Southwest University Chongqing 400716

China

E-mail: hcui@swu.edu.cn, hongjuan.cui@gmail.com

Yongzhong Wang, PhD

Department of Radiology

Fourth Hospital of Hebei Medical University Shijiazhuang, Hebei Province 050011

China

E-mail: 929275222@qq.com

Abbreviations Used
BrdU $=$ 5-bromo-2-deoxyuridine
DMSO $=$ dimethyl sulfoxide
MTT $=3$ - $(4,5$-dimethylthiazol-2-yl $)-2,5$-diphenyl
tetrazolium bromide
PBS $=$ phosphate-buffered saline
PI $=$ propidium iodide
PTCH $=$ Patched 1
RT-PCR $=$ reverse-transcription polymerase chain reaction
SHH $=$ Sonic Hedgehog
SMO $=$ Smoothened

